\title{
FIXED POINT FREE INVOLUTIONS ON HOMOTOPY SPHERES
}

BY W. BROWDER AND G. R. LIVESAY ${ }^{1}$

Communicated by J. Milnor, November 9, 1966

1. Introduction and statements of theorems. Let $T: \Sigma^{n+1} \rightarrow \Sigma^{n+1}$ be a smooth ${ }^{2}\left(C^{\infty}\right)$ fixed point free involution on a smooth manifold, $\Sigma^{n+1}$, homeomorphic to the $(n+1)$-sphere, $S^{n+1}$. We wish to consider the following problem: does there exist an $n$-sphere, $S^{n}$, smoothly imbedded in $\Sigma^{n+1}$ such that $T S^{n}=S^{n}$ ? If such an $S^{n}$ exists, we will say that $\left(T, \Sigma^{n+1}\right)$ desuspends to $\left(T \mid S^{n}, S^{n}\right)$ and that $\left(T \mid S^{n}, S^{n}\right)$ suspends to $\left(T, \Sigma^{n+1}\right)$. We claim (proofs are to appear later):

Theorem 1. If $n \geqq 5$ is odd, then $\left(T, \Sigma^{n+1}\right)$ desuspends to $\left(T \mid S^{n}, S^{n}\right)$ for some $T$-invariant $S^{n} \subset \Sigma^{n+1}$.

If $n$ is even, there are obstructions to desuspending $\left(T, \Sigma^{n+1}\right)$. There is a bilinear form, $B(x, y)$ defined on a certain subgroup of $H_{*}(M)$, where $\Sigma^{n+1}=A \cup T A, A$ and $T A$ are compact submanifolds of $\Sigma^{n+1}$ with smooth boundary, and $\partial A=\partial T A=A \cap T A=M$. If $n \equiv 2(\bmod 4)$, then $B$ is symmetric, and its signature, $\sigma\left(T, \Sigma^{n+1}\right)$ is determined by $\left(T, \Sigma^{n+1}\right)$. If $n \equiv 0(\bmod 4)$, then $B$ is skew-symmetric. Furthermore, if $n=4 k$, there is a map $\psi_{0}: H_{2 k}\left(M ; Z_{2}\right) \rightarrow Z_{2}$ such that $\psi_{0}(x+y)=\psi_{0}(x)+\psi_{0}(y)+B_{2}(x, y)$, where $B_{2}$, defined on a subgroup of $H_{2 k}\left(M ; Z_{2}\right)$, corresponds to $B$, defined on a subgroup of $H_{2 k}(M)$. The Arf invariant, $c\left(T, \Sigma^{n+1}\right),[1],[4]$, corresponding to $\psi_{0}$ and $B_{2}$, depends only on $\left(T, \Sigma^{n+1}\right)$. Regarding these invariants, we have

Theorem 2. If $n \equiv 2(\bmod 4)$ and $n>5$, then $\left(T, \Sigma^{n+1}\right)$ can be desuspended to $\left(T \mid S^{n}, S^{n}\right)$ if and only if $\sigma\left(T, \Sigma^{n+1}\right)=0$.

THEOREM 3. If $n \equiv 0(\bmod 4)$ and $n>4$, then $\left(T, \Sigma^{n+1}\right)$ can be desuspended to $\left(T \mid S^{n}, S^{n}\right)$ if and only if $c\left(T, \Sigma^{n+1}\right)=0$.

At present, we have no example of $\left(T, \Sigma^{n+1}\right)$ for which either $c\left(T, \Sigma^{n+1}\right) \neq 0$ for $n \equiv 0(\bmod 4)$, or $\sigma\left(T, \Sigma^{n+1}\right) \neq 0$ for $n \equiv 2(\bmod 4)$. An interesting example to study in connection with the possibility of

1 The authors were partially supported by contracts GP 2425 and GP 3685 , respectively, with the National Science Foundation.

${ }^{2}$ The results hold equally in the piecewise linear category with little change in the proofs. 
a nonzero Arf invariant is the nonstandard involution of Hirsch and Milnor on $S^{5}$, [3]. However, even if the Arf invariant is zero, our methods do not give a desuspension of this involution to the 4 -sphere, because of the usual difficulties of finding a basis for $H_{2}\left(M^{4}\right)$ represented by imbedded spheres.

Regarding the uniqueness of the desuspension, we have

Theorem 4. If $n \geqq 4$ is even, and $\left(T, \Sigma^{n+1}\right)$ desuspends to $\left(T \mid S_{0}^{n}, S_{0}^{n}\right)$ and to $\left(T \mid S_{1}^{n}, S_{1}^{n}\right)$, then $\left(T \mid S_{0}^{n}, S_{0}^{n}\right)$ and $\left(T \mid S_{1}^{n}, S_{1}^{n}\right)$ are equivariantly concordant in $\Sigma^{n+1} \times I$.

We say that $\left(T_{0}, S_{0}^{n}\right)$ and $\left(T_{1}, S_{1}^{n}\right)$ are concordant if there exists a fixed point free involution $T: S^{n} \times I \rightarrow S^{n} \times I$, where $I=[0,1]$, such that $T\left(S^{n} \times 0\right)=S^{n} \times 0$, and equivariant diffeomorphisms $i_{0}:\left(T_{0}, S_{0}^{n}\right)$ $\rightarrow\left(T \mid S^{n} \times 0, S^{n} \times 0\right)$ and $i_{1}:\left(T_{1}, S_{1}^{n}\right) \rightarrow\left(T \mid S^{n} \times 1, S^{n} \times 1\right)$. If $T: \Sigma^{n+1}$ $\rightarrow \Sigma^{n+1}$ is a smooth, fixed point free involution, then $\left(T_{0}, S_{0}^{n}\right)$ and $\left(T_{1}, S_{1}^{n}\right)$ are concordant in $\Sigma^{n+1} \times I$ if they are concordant, and $\left(T, S^{n} \times I\right)$ is equivariantly imbedded in $\left(\bar{T} \times 1, \Sigma^{n+1} \times I\right)$ with $S^{n} \times 0 \subset \Sigma^{n+1} \times 0, S^{n} \times 1 \subset \Sigma^{n+1} \times 1$. If $n>4$ is odd, the signature and Arf invariant, which appeared as obstructions to desuspending $\left(T, \Sigma^{k}\right)$, now appear as obstructions to obtaining a concordance in $\left(T \times 1, \Sigma^{n+1} \times I\right)$ between two given desuspensions, $\left(T \mid S_{0}^{n}, S_{0}^{n}\right)$ and $\left(T \mid S_{1}^{n}, S_{1}^{n}\right)$. If $S_{0}^{4 k-1}$ and $S_{1}^{4 k-1}$ are two invariant spheres in $\left(T, \Sigma^{4 k}\right)$, then $\sigma\left(T, \Sigma^{4 k}, S_{0}^{4 k-1}, S_{1}^{4 k-1}\right)$, the signature of a certain bilinear form, is defined. We then have

TheOREM 5. $S_{0}^{4 k-1}$ and $S_{1}^{4 k-1}$ are concordant in $\left(T \times 1, \Sigma^{4 k} \times I\right)$ if and only if $\sigma\left(T, \Sigma^{4 k}, S_{0}^{4 k-1}, S_{1}^{4 k-1}\right)=0$. In particular, if $\sigma=0$, then $\left(T \mid S_{0}^{4 k-1}, S_{0}^{4 k-1}\right)$ and $\left(T \mid S_{1}^{4 k-1}, S_{1}^{4 k-1}\right)$ are equivariantly diffeomorphic.

Now suppose $S_{0}^{4 k+1}$ and $S_{1}^{4 k+1}$ are invariant spheres in $\left(T, \Sigma^{4 k+2}\right)$. Then $c\left(T, \Sigma^{4 k+2}, S_{0}^{4 k+1}, S_{1}^{4 k+1}\right)$, an Arf invariant, is defined.

TheOREM 6. $S_{0}^{4 k+1}$ and $S_{1}^{4 k+1}$ are concordant in $\left(T \times 1, \Sigma^{4 k+2} \times I\right)$ if and only if $c\left(T, \Sigma^{4 k+2}, S_{0}^{4 k+1}, S_{1}^{4 k+1}\right)=0$.

Corollary. If $n \equiv 1(\bmod 4)$, there are at most two invariant $n$ spheres in $\Sigma^{n+1}$, up to equivariant diffeomorphism.

It is planned to present detailed proofs later. We will, however, indicate briefly some of the ideas involved.

2. Characteristic submanifolds. Let $T: \Sigma^{n+1} \rightarrow \Sigma^{n+1}$ be a fixed point free smooth involution. A characteristic submanifold $M^{n} \subset \Sigma^{n+1}$ is an $n$-manifold smoothly imbedded in $\Sigma^{n+1}$ such that $\Sigma^{n+1}=A \cup T A$ with $A \cap T A=M^{n}$. We have a commutative square 


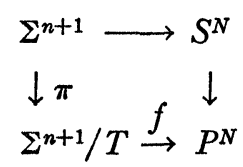

where $N$ is large, $P^{N}$ is a real projective $N$-space, and $f$ classifies the principal $Z_{2}$-bundle $\Sigma^{n+1} \stackrel{\pi}{\rightarrow} \Sigma^{n+1} / T$. By making $f$ transverse-regular [5] on $P^{N-1}, \pi^{-1} f^{-1} P^{N-1}$ will be a characteristic submanifold. It is easy to see that all characteristic submanifolds arise in this way. Any two characteristic submanifolds are equivariantly cobordant in $\left(T \times 1, \Sigma^{n+1} \times I\right)$. (The definition is analogous to that of concordance in $\Sigma^{n+1} \times I$.) It is this fact that makes the signature and Arf invariant independent of the choice of characteristic submanifold.

3. The signature and Arf invariant. Let $M$ be a characteristic submanifold in $\Sigma^{n+1}$. Then $\Sigma^{n+1}=A \cup T A$ with $A \cap T A=M$. We have the Mayer-Vietoris sequence

$$
\cdots \rightarrow H_{p+1}\left(\Sigma^{n+1}\right) \rightarrow H_{p}(M) \stackrel{\left(i_{A}, i_{T A}\right)}{\longrightarrow} H_{p}(A) \oplus H_{p}(T A) \rightarrow H_{p}\left(\Sigma^{n+1}\right) \rightarrow \cdots
$$

If $n=2 k, k>0$, and $p=k$, this becomes

$$
0 \rightarrow H_{k}\left(M^{2 k}\right) \stackrel{\left(i_{A}, i_{T A}\right)}{\longrightarrow} H_{k}(A) \oplus H_{k}(T A) \rightarrow 0
$$

and so $H_{k}\left(M^{2 k}\right)=\operatorname{ker} i_{A} \oplus \operatorname{ker} i_{T A}$, and $T * \operatorname{ker} i_{A}=\operatorname{ker} i_{T A}$. Since $M^{2 k} C \Sigma^{2 k+1}, M$ is orientable, and a bilinear form $B(x, y)=x \cdot T * y$ is defined, for $x$ and $y$ in ker $i_{A}$. Since $T$ preserves orientation in $\Sigma^{2 k+1}$, it reverses orientation in $M^{2 k}$, and the bilinear form $B$ is symmetric (skew-symmetric) when the intersection form $x \cdot y$ is is skew-symmetric (symmetric). Therefore, given $\left(T, \Sigma^{n+1}\right)$ and a characteristic submanifold $M^{n}$, if $n \equiv 2(\bmod 4)$, the signature of the form $B(x, y)$ is determined, and turns out to be independent of the choice of characteristic submanifold. The reason for considering the signature of $B$ is the following. If $x \in \operatorname{ker} i_{A} \subset H_{k}\left(M^{2 k}\right)$, and $M^{2 k}$ is $(k-1)$-connected, (which we achieve by exchanging handles between $A$ and $T A$ ) then $x$ is represented by an imbedded $S^{k} \subset M^{2 k}$, which bounds a cell $D^{k+1} \subset A$. (This statement may be false for $k=3,[2]$, but a different argument applies in this case.) Supposing $M^{2 k}$ is totally geodesic near $D^{k+1}$, we take a tubular neighborhood $N$ of $D^{k+1}$, replace $A$ by $A-N$, and replace $T A$ by $T A \cup \bar{N}$. This will reduce the rank of $H_{k}(M)$. However, $(A-N) \cap(T A \cup \bar{N})=M^{\prime}$ is no longer $T$-invariant. We may obtain an invariant $M^{\prime}$ if we replace $A$ by $(A-N) \cup T \bar{N}$, and replace $T A$ by $(T A \cup \bar{N})-T N$. However, to do this we need $S^{k} \cap T S^{k}=\phi$. It is to accomplish this that we need $\sigma=0$ 
when $k$ is odd and $c=0$ when $k$ is even. The distinction between the two cases arises since if $S^{k}$ and $T S^{k}$ intersect transversally in $M^{2 k}$ at a point $p$ with intersection number 1 , then they intersect at $T p$ with intersection number $(-1)^{k+1}$.

The cohomology operation, $\psi(x)$, used to define the Arf invariant, merely serves to count, $\bmod 2$, the number of pairs $(q, T q)$ of points in $S^{p} \cap T S^{p}$, where $S^{p}$ represents the Poincaré dual of $x$, and the intersection is transverse.

\section{REFERENCES}

1. C. Arf, Untersuchungen ïber quadratische Formen in Körpern der Charakteristik 2, J. Reine Angew. Math. 183 (1941), 148-167.

2. A. Haefliger, Knotted (4k-1)-spheres in 6k-space, Ann. of Math. 75 (1962), 452-466.

3. M. W. Hirsch and J. W. Milnor, Some curious involutions of spheres, Bull. Amer. Math. Soc. 70 (1964), 372-377.

4. M. A. Kervaire and J. W. Milnor, Groups of homotopy spheres. I, Ann. of Math. 77 (1963), 504-537.

5. R. Thom, Quelques propriettes global des variettes differentiables, Comment. Math. Helv. 28 (1954), 17-86.

\section{Institute for Advanced Study, Princeton University AND CORNELl University}

